\title{
L'histoire de la Loire de la Guerre de cent ans à nos jours
}

\author{
par A. Poitrineau \\ Professeur émérite à l'Université Blaise Pascal, Clermont-Ferrand II
}

\section{E UN FLEUVE ROYAL}

L'histoire de la Loire à l'époque moderne est indissociable de celle des hommes qui ont vécu près d'elle, autour d'elle, et bien souvent d'elle ou grâce à elle. Ce fleuve qui, avec ses affluents, arrose le sixième de la France, a été l'un des acteurs majeurs de la guerre de cent ans, qui a scellé pour des siècles le devenir du pays. On s'est battu sur ses rives, autour de ses ponts, pour le contrôle des riches provinces qui restaient au « Roi de Bourges » et que convoitaient les envahisseurs anglais. Les rois de France ont levé de lourds impôts, sous le nom de «cloison de Loire " pour élever ou renforcer sur la ligne d'eau rudement disputée, les forteresses salvatrices qui en gardent encore le cours actuellement. C'est sur la Loire que s'est scellé le sort du royaume, alors même que Charles, duc d'Orléans, le « gentil poète » en célébrait avec nostalgie, le charme changeant.

Après Charles VII, les Rois de France ont fait de la vallée de la Loire leur résidence favorite et l'ont semée de superbes résidences de plaisance, ces joyaux architecturaux qui restent de nos jours une des plus sûres valeurs touristiques de notre pays. Au temps où Louis XI faisait de Tours sa véritable capitale, au temps où les cours brillantes des Valois y nomadisaient de château en château, de chasse en chasse, la civilisation française atteignait un sommet grâce à des écrivains, des artistes, et des artisans qui, souvent, étaient des Ligériens, marqués par le génie de leurs terroirs.

Cette floraison architecturale et artistique n'a été possible que grâce à l'importance alors revêtue par la Loire comme voie de communication et donc comme artère nourricière pour la France moyenne. Cette importance perdura pendant les siècles suivants. Ce n'est pas un hasard, si les guerres de religion et les troubles des minorités royales eurent la vallée de Loire comme théâtre d'opérations privilégié. Pour le futur empereur germanique Joseph II, visitant incognito la France à la veille de la Révolution, la Loire restait « la veine cave du royaume».

Français ou étrangers, pendant un demi-millénaire, les voyageurs furent légion, qui décrivirent, chacun selon son tempérament, les aspects multiples d'un dynamisme induit et entretenu par un fleuve multiforme, puissant et capricieux, séduisant et inquiétant.

\section{II 圆 LA LOIRE EXALTÉE}

\subsection{Des bateaux et des hommes}

Car la Loire «porte bateau ». Chemin qui marche, elle constitue une rocade naturelle pour la navigation et les échanges. Tout un peuple de pêcheurs, de meuniers et de mariniers vit sur l'eau et par l'eau. Les uns exploitent le fleuve poissonneux, au moyen de pièges ingénieux, de filets, et de techniques séculaires. les autres se servent de l'énergie hydraulique pour, à grand renfort de "moulins pendus " sous les arches des ponts, ou de "moulins flottants» sur ancres et roulis - sans parler des moulins à cavier qui, par grappes occupent les coteaux bordiers - travailler les grains et les chanvres de la vallée. Quant aux mariniers, " compagnons de rivière ", ils animent en permanence le fleuve et ses abords. Car les rives de la Loire et de ses grands affluents fourmillent de "ports", où abordent les bateaux, où se pressent les entrepôts de marchandises, à proximité des bourses de fret que sont les « auberges de la marine».

Ces bateaux de Loire, ... si divers, et pourtant si typés ! Dès le Moyen Age, c'en est fini des pirogues monoxyles qui ont sillonné le fleuve dans les temps très anciens, et dont on extrait des sablières un spécimen de temps à autre. Mais les bateaux à piautres - la piautre est le gouvernail particulier aux bateaux ligériens, caractérisé par son axe oblique et sa très grande taille - y circulent en masse, d'Orléans à Nantes; ils profitent des vents de galerne qui gonflent leurs voiles rectangulaires pour remonter le courant, et s'aident de celui-ci pour le descendre. Ce sont les gros chalands de chêne chevillé, avec à leur suite les tirots aux voiles décroissantes, formant de véritables trains, les saumuroises ou les toues, de moindre échantillon, et les modestes futureaux; au passage des ponts, on abaisse leurs mâts à l'aide d'un gros treuil horizontal, le guindas. Spécialisés sont les bateaux pour passagers ou cabanes, portant l'habitacle toituré dans lequel se tiennent les passagers, ou la bascule, bateau percé qui sert de vivier pour le transport des poissons. Sur tout le 
cours de la Loire et de l'Allier, on trouve une autre catégorie de bateaux, les sapines, qui tirent leur nom des bois résineux dont elles sont faili : on les nomme aussi, selon leur origine, des salembarles (Saint-Rambertes), des auvergnates, des roannaises. "Déchirés » dès leur arrivée à destination, ces bateaux d'un seul voyage, ne circulent qu'en suivant le fil de l'eau, à la descente; ce sont des caisses longues et étroites, faites de pièces chevillées, avec un arrière en tableau et une proue en sifflet. Pas de voiles: les mariniers les dirigent en pesant sur les énormes « bâtons de marine ", qu'ils plantent dans le fond et appuient sur les arronçoirs des coques. Enfin, troisième catégorie de bateaux - à fond plat, comme les deux autres - les nombreux bacs, généralement à cordes, qui permettent aux homimes, au bétail, et parfois aux voitures le passage d'une rive à l'autre. Les passeurs qui les mènent assurent un service régulier et payant; sur eux repose la vie de relation entre les rives de chaque cours d'eau. Pour achever le tableau, les radeaux, organisés en trains de bois, acheminent indolemment vers l'aval les mâts de marine et les bois, équarris ou non, tirés de forêts du haut pays.

La navigation, compliquée par les multiples péages exigés sur tous les cours d'eau du système, est rendue délicate par le régime irrégulier des eaux, par les épaves, par le passage des ponts. Elle est affaire de tours de main et de techniques mises au point par des générations de mariniers et de pêcheurs attachés de père en fils à leur métier. Ces gens de rivière, querelleurs, férus de leurs traditions et débrouillards, furent « classés » par ordonnance royale de 1780, et astreints dès lors à servir dans la marine de guerre par roulement, en temps de conflit, ce qui valut à nombre d'entre eux de voir du pays.

\subsection{Les infrastructures}

Il serait inexact de prétendre que les premiers travaux d'aménagement de la Loire n'ont été entrepris qu'après 1815. De fait, les nœuds de trafic les plus actifs bénéficient de notables améliorations d'infrastructure, bien avant le XVIII ${ }^{\mathrm{cm}}$ siècle.

Sur la Loire, et autour d'elle, les hommes ont beaucoup travaillé. Travail de Pénélope, bien souvent. Vers 1720, l'ingénieur du Roi Nicolas Poictevin, chargé d'une enquête sur tout le secteur fluvial situé en aval d'Orléans, constate que le tiers des ponts existants est à démolir et reconstruire ; les routes terrestres qui franchissent la ligne d'eau, par des gués problématiques ou sur des ouvrages disparates (des arches de pierre, démolies par les crues, étant remplacées par des tabliers de bois) sont en très mauvais état, et bien souvent impraticables. Dès le Moyen Age, on a commencé à édifier des levées, ou «turcies " pour protéger les villages et les riches terroirs des vals contre les colères subites du fleuve. Au temps de Colbert, ces travaux, et bien d'autres qui ont trait à la rectification du cours de la Loire et à l'approfondissement de son chenal au profit de la navigation, s'amplifient. Le désenclavement du bassin est mené à bien par la construction, de 1604 à 1642 du canal de Briare, prouesse technique pour l'époque, puis par celle du canal d'Orléans, achevé en 1692.

Désormais, par le Loing, la batellerie de Loire peut déboucher sur la Seine et approvisionner Paris. Et grâce à ce complexe bien équipé en écluses, en magasins, en caves et en étangs régulateurs, la compagnie du canal de Briare devient pour longtemps une affaire des plus lucratives. Sur le haut fleuve, c'est entre 1700 et 1825 qu'une série d'aménagements - dont les célèbres digues de La Roche et de Pinay - améliore le régime des eaux. Entre 1831 et 1838 , enfin, on creuse le canal de Roanne à Digoin pour favoriser l'écoulement par eau des charbons locaux.

\subsection{Des « ports chargeables »}

Avant le XIX ${ }^{\text {eme }}$ siècle, la notion de port doit, en matière fluviale, être prise dans un sens restrictif. L'infrastructure indispensable au chargement, au déchargement, à l'entretien et au séjour des bateaux, tant du type «chaland » que du genre "sapinière ", se borne à très peu de choses. Mais les "ports chargeables » du bassin ligérien forment un chapelet qui s’égrène le long du cours navigué de la Loire et de ses affluents : relativement rares vers I'amont, à proximité des terminus de navigation, ils s'agglomèrent parfois en grappes denses dans les bas pays.

Survivance du Moyen Age, nombre de ports ligériens se réduisent encore à la fin de l'Ancien Régime, à des « chantiers ", à peine gardés symboliquement. En 1789, dans leur cahier de doléances, les voituriers par eau d'Angers affirment que tous les ports de la ville restent exposés aux vols et aux inondations. Au XVII ${ }^{\mathrm{eme}}$ siècle, le port de Poitiers, sur le Clain, près de la porte de Paris, n'est marqué que par quelques anneaux de fer scellés dans des rochers, pour l'amarrage des bateaux. Ne réclamant guère d'investissements initiaux, ces ports occupent des sites favorables : abri d'une île, comme à Roanne, anse ou confluent de deux cours d'eau comme Candè. Le port de La Charité, "l'un des plus fréquentés de la Loire " vers 1744, n'est que «le chantier naturel de la rivière, fort sujet à être dégradé par les crues et les glaces,... trop étroit pour contenir la quantité de marchandises différentes qu'on y dépose pour les embarquer, et trop bas pour que ces marchandises puissent y rester sans risques lorsqu'il arrive des crues subites, pendant la nuit surtout "; on se borne - réparations dérisoires - à jeter du gravier ou de la terre pour combler les brèches qui se forment dans la rive: les murs anciens de la ville, le long du port, tombent en ruines et risquent d'être renversés et emportés, au grand dam des maisons adjacentes qui suivraient inéluctablement. D'où la nécessité urgente d'un "mur de quay" construit à chaux et à sable avec "des rampes de distance en distance». A Selles, sur le Cher, le port dit "de la Pêcherie" ne consiste qu'en un "talus à usage de port », édifié à la diable, peu élevé, et incapable même de défendre l'agglomération contre une crue sérieuse de la rivière. Les réparations et constructions de bateaux y nuisent au chargement et déchargement, et tous les bestiaux de la localité flottant tout proches, qui créent des courants dangereux.

Tours, à la fin du XVIII ème siècle, a ses ports nombreux et médiocres, mais actifs, tant sur la rive droite, au Portillon, paroisse de Saint-Cyr, et au Port Barillet (paroisse de SaintSymphorien), que sur la rive gauche, avec le Port Feu Hugon, le port de l'Echallerie (paroisse Saint-Julien), le port de la Poissonnerie (paroisse Saint-Martin), le Port Bretaigne au ruau Saint-Anne (paroisse Notre-Dame de La Riche) ou encore, à proximité, le Port de la Clarté (Saint-Pierre des Corps).

Quant aux «ports " de Saumur, ils paraissaient en 1777, à en croire les marchands drapiers de la ville, assez misérables. Celui de la Bilange est comblé par les cailloux, les blocs de tuffeau, la terre des déblais que l'on y jette quotidiennement; le port au bois est encombré de tuffeaux à demi immergés qui empêchent les bateaux, au risque de se perdre, d'aborder; et ceux de Saint-Michel, des Ardillliers et du faubourg de Fenet, mal tenus, aux rives indûment plantées d'arbres par des particuliers, obstrués par des bateaux en radoub ou en cours de déchirage ou des marchandises.

Sur la Sarthe et sur la Mayenne, les ports ne consistent, eux aussi, qu'en des rives en plan incliné sur lesquelles on tire les bateaux au sec; ainsi le port d'Arnage. Sur le canal de Briare, "deux parties de près dans l'île de Rogny " constituent une aire de stockage des bois empilés, descendus par flottage de Saint-Fargeau. 
Tous ces pseudo-ports, qu'on nomme parfois cales, sont périodiquement balayés par les crues qui emportent marchandises, bateaux et entrepôts - lorsqu'il en existe sous forme de baraques. Enormes sont par exemple les dommages à Montreuil-Bellay, sur le Touet navigable, en novembre 1771 , ou à Puy-Guillaume en novembre 1790 . De plus, les déplacements fréquents du chenal de la Loire obligent souvent les communautés d'habitants à changer l'emplacement de leurs ports : ainsi, celui de Montsoreau, pendant longtemps sur la rive gauche du fleuve et contigu au village, est-il en 1830 de l'autre côté de l'eau, sur la rive droite. Un rapport postérieur à 1844 établit qu' « actuellement, la plupart des ports de la Loire sont en été ensablés à une très grande distance en avant des rives, de manière à ne pouvoir même y aborder avec un simple batelet tirant à peine 6 pouces d'eau ".

L'incommodité et la précarité de ces infrastructures, la volonté d'instaurer sur la Loire "une navigation réglée » plus apte à lutter contre la concurrence croissante de la voie de terre (rouliers et diligences), incitent les usagers et les professionnels du transport par eau à réclamer des "ports construits ». Aussi, dans le cadre d'une politique d'équipement du pays favorable au développement des communications, les villes ligériennes les plus importantes en matière de navigation fluviale, puis, progressivement de moindres centres de batellerie, vont-ils être pourvus de quais maçonnés avec chaussées pavées, de "gares d'eau », de calesabreuvoirs - bref de ces agencements permanents bâtis en dur qui peuvent faciliter la vie professionnelle des gens de rivière et des débardeurs, aussi bien qu'accélérer la rotation des marchandises.

A Nantes, à la charnière de la navigation de mer et de la batellerie fluviale, un vaste projet de construction de quais le long de la Loire et de modernisation des ponts toujours menacés de ruine et dangereux pour les chalands ou sapines, avait été établi vers 1724, puis, au terme d'une longue enquête, exécuté en 1740-42. A Montjean, sur la Loire angevine, un petit complexe portuaire fut mis en service à partir de 1785 : il était constitué par le quai d'accostage dit «du saumon", et par une grève d'échouage en plan incliné munie de deux treuils à bras pour hisser les bateaux à sec : un type de machine qui est alors en service - l'iconographie le prouve - tout le long du fleuve. A Blois, en abattant une rangée de maisons, on établit un quai au faubourg de Vienne. A Nevers, pour desservir l'établissement métallurgique de La Chaussade, acquis par le Roi, et qui travaille pour la flotte de guerre, on établit vers 1782 un vaste quai, dit «de Médine», muni d'escaliers ménagés dans le perré, pour permettre aux manœuvres de transférer plus commodément de terre aux bateaux ou vice-versa, le charbon et les énormes ancres de marine forgées sur place ; une vaste gare à bateaux «pour qu'ils soient absolument en sûreté lors des inondations ou des glaces" y est par ailleurs mise à la disposition des "bateaux du commerce en relâche". En même temps, on achève des travaux destinés à rectifier « de la manière la plus avantageuse pour la navigation ", la trajectoire des courants qui se présentaient obliquement par rapport au pont de Nevers, et jetaient les bateaux sur ses piles. A Cosne, un quai de pierre a déjà été mis en service en 1779, pour faire desservir les forges locales, par la marine de Loire. Tandis que des projets tendant à l'établissement de quais et de ports modernes sont mis à l'étude à Ancenis et à Ingrandes, on établit des plates-formes à rampes pavées et inclinées aux Roziers, à Sully, aux Ponts-de-Cé, où les Hollandais chargent les vins d'Anjou, - à Meung, Saumur, Montsoreau, Amboise, Jargeau... Pour parer au péril fréquent des glaces flottantes et des embâcles, on installe des garesabris pour les bateaux, dans les boires de la Loire moyenne, à l'aide d'estacades en charpente. A Châteauneuf, le duc de Penthièvre, seigneur du lieu et philantrope notoire, finance le grand port d'aval, seul achevé à la Révolution, et le petit port d'amont.

\subsection{La splendeur d'Orléans}

Très vite, Orléans, placé au centre du dispositif ligérien, tient le rang d'un emporium qui commande tout le système économique du bassin.

Bénéficiant d'une rente de situation, la vieille cité des Carnutes joue, parmi les ports fluviaux du système ligérien, un rôle d'entrepôt régulateur. Dès le $X V^{\text {ème }}$ siècle, sa fonction commerciale éminente est attestée par des auteurs comme Louis Trippault :

"Icelle ville a aux pieds de ses murailles la rivière de Loire, fleuve excellent et bien commode, par lequel y sont de divers endroits, voicturés et conduictes plusieurs riches marchandises tirées de aucunes bonnes villes de France et de païs étrangers. " D'ailleurs, du XIII ${ }^{\text {ème }}$ siècle à 1772 , Orléans demeure le siège social de la séculaire compagnie des «Marchands fréquentans la riviére de Loyre et fleuves descendant en icelle. " Des assemblées de la société, qui s'y tiennent tous les trois ans, à la maison de ville, émanent des décisions qui engagent l'ensemble de la navigation dans tout le bassin ligérien. Les modalités d'une surveillance opiniâtre, poursuivie dans chacune des 22 circonscriptions territoriales (ou «détroits») par un délégué nommé par les gens de rivière, tendent à maintenir ou si possible à améliorer le trafic dont Orléans, à la charnière des « pays hauts " de la Loire supérieure et des «bas pays " centrés sur les vals, est la plaque tournante. Les marchands fréquentans, entrepreneurs en transports et négociants par eau, profitent de privilèges octroyés par le pouvoir royal et régulièrement renouvelés. Ils ont le droit de lever une contribution sur les usagers de la voie fluviale, la boette, et de porter directement leurs procès devant le Parlement de Paris. Jusqu'au milieu du XVIII ${ }^{\text {èmc }}$ siècle, leur compagnie veille au bon état de l'artère fluviale et de ses principaux affluents, dont ils mettent en adjudication l'entretien et le nettoiement régulier. Muni de la personnalité civile, le puissant consortium des marchands fréquentans fait supprimer ou modérer les péages abusifs, et établir des tarifs fixes pour ceux qui sont maintenus.

La ville vit de ses ports. Son vieux pont sépare emblématiquement deux domaines fluviaux fortement contrastés; à l'aval, le port de Recouvrance et ses annexes d'Avenon se consacrent à la navigation d'en bas, avec ses chalands vergés (mâtés), ses tirots, allèges et autres bateaux à deux levées; à l'amont, le port de la Poterne et ses abords grouillent de sapines de toutes tailles venues au fil du courant du Forez, de l'Auvergne et du Nivernais, tandis que sur la rive gauche, le Portereau est le port du bois, où abordent les radeaux flottés descendus du massif central.

Port intérieur et port de contact, Orléans est aussi un port d'entrepôt et un centre de redistribution de produits d'autant plus variés qu'ici affluent des marchandises expédiées de Nantes, de Lyon, de Paris, et, via ces villes de grand commerce, de contrées très lointaines, situées en Europe ou hors d'Europe.

Au siècle des Lumières, le vieux port d'Orléans, tel que le décrit Daniel Polluche en 1734, est remplacé par un nouveau port situé un peu plus à l'aval tandis que la démolition des remparts croulants sur le front de Loire permet l'aménagement de quais spacieux, pourvus de vastes terre-pleins et de rampes en plan incliné; le «duit » ou digue submersible qui canalise le courant majeur est établi ; Orléans se donne une large et belle façade sur la Loire nourricière ; un état des lieux dressé par le bureau des finances et daté de 1780 fournit un bilan balancé des transformations récentes:

«...la ville est divisée en son centre par la rue royalle, qui aboutit à un pont construit depuis peu d'années par la munificence du feu Roy; du côté droit en descendant la Loire, on vient de terminer des quays aussi majestueux que nécessaires pour le commerce du couchant avec les villes de Blois, Tours, Saumur; Nantes et les provinces adjacentes. Du côté gauche en remontant la Loire est située la partie com- 
merçante avec les villes supérieures jusqu'à Lyon et même jusqu'à Marseille à cause de la guerre (guerre contre l'Angleterre). De ce côté, il a été fait il y a plusieurs années des quays appellés de la Tourneuve et de la Poterne, pour le débarquement de toutes les marchandises du Levant. Mais ces quays ont été interrompus prés de l'ancien palais des Rois d'Orléans appelé le Châtelet où les différents tribunaux rendent aujourd'huy la justice, parce que ces murs étaient baignés par la riviére. "

Et de conclure en réclamant, avec les marchands, que les rues étroites et rapides qui dévalent vers le quai de la Poterne soient élargies et aplanies, et que les matériaux de démolition qui proviendront de ces travaux voyers soient entassés au bord de la Loire devant le Châtelet, pour établir une communication d'autant plus indispensable entre les ports du Ponant et du Levant que l'encombrement y est extrême : plus de 300 voitures - chars, tombereaux, fardiers et charrettes s'y affairent chaque jour ! Programme qui ne sera mené à bien qu'après 1815 , lorsque de grands travaux doteront Orléans d'une vaste "gare d'eau ».

Cet effort porte ses fruits. A la veille de la Révolution, l'anglais Arthur Young, observateur souvent malveillant, note l'activité de la ville et du port:

"Il y a sur le quai beaucoup de chalands et de bateaux, construits sur le fleuve dans le Bourbonnais... chargés de vin, de bois, d'eau de vie et autres marchandises ; à l'arrivée à Nantes, ils sont démolis et vendus avec la cargaison. Un grand nombre de ces bateaux est en sapin. Un bateau va d'ici à Nantes quand six personnes demandent le passage: chacune doit payer un louis d'or: on fait escale la nuit et il faut quatre jours et demi pour atteindre Nantes. La rue principale, qui conduit au pont, est belle; toute la ville est active et animée car le commerce y est important... »

Vers 1840 , la prospérité de la navigation ligérienne atteint un sommet. Ce n'est pourtant que son chant du cygne. Le mouvement du port d'Orléans frise les trente bateaux par jour ; parfois 300 sapines ou chalands s'y trouvent à la fois, et les diverses compagnies de "paquebots " à vapeur se disputent les passagers sur tout le cours du fleuve; mais le trafic n'est déjà plus ce qu'il était un demi-siècle plus tôt, quand selon la municipalité, l'activité fluviale faisait vivre à Orléans 800 familles de mariniers, et par ricochet des milliers de débardeurs, de tonneliers, charrons, bourreliers, charretiers, cordiers et charpentiers occupés aux manipulations, au transbordement, au conditionnement des marchandises, et à la construction ou à l'entretien des bateaux... quand le transport et l'hébergement des voyageurs; des commissionnaires et des marchands étrangers y faisaient vivre quantité d'aubergistes et de cabaretiers.

\subsection{Trafics...}

Sur la Loire et ses affluents circulaient les produits les plus variés, qui nous sont connus par les comptes de péages parvenus jusqu'à nous. A la remontée, c'était le sel de l'Océan, qui gagnait par lourds convois hâlés, sévèrement surveillés, les greniers à sel des provinces du Centre. Et, venant de Nantes, le poisson, salé, fumé ou frais, les huîtres, les bois exotiques, le tabac - ainsi, au XVIII ${ }^{\text {ème }}$ siècle, que le sucre " terré » des Isles, à destination des raffineries de Saumur, de Blois ou d'Orléans. En sens inverse, c'étaient les marchandises embarquées à Roanne, le port de Lyon, et parmi elles, celles qui venaient des rives de la Méditerranée par le Rhône, telles que l'huile d'olive, le savon, les anchois, les agrumes.

Le vin de Touraine, d'Anjou et du Pays nantais va à Nantes par eau, à destination des Antilles ; celui de l'Orléanais et du Blésois se dirige, par la Loire et le canal de Briare, vers les cabarets parisiens. La houille en provenance de l'Auvergne, du Bourbonnais et du Forez devient après 1720 un fret essentiel. Les fruits à la saison, les poissons d'eau douce, le bois, les fers et produits métallurgiques du Berry et du Nivernais, mais aussi les céréales, les chanvres, les ardoises, voire aux XVI ${ }^{\text {ème }}$ et XVII ${ }^{\text {ème }}$ siècles les soieries de Lyon, les livres qui y sont imprimés, le papier et les armes de Saint-Etienne empruntent également la voie d'eau. Non sans risques, car les naufrages ne sont pas rares. Mais la voie de terre est aussi lente et n'est pas plus sûre..

Les hommes - moines, soldats, princes, " escholiers » et marchands - et les femmes - telle madame de Sévigné empruntent également la voie d'eau, le long de laquelle ils trouvent des auberges réputées, pour la nuitée et la table.

La Loire et ses affluents les plus importants constituent de la sorte un système de communications coordonnées qu'exploite, bon an mal an, une poussière d'entreprises privées.

\section{III $\mathbf{n}$ LA LOIRE DÉSERTÉE}

La crise s'annonce à la veille de la Révolution ; l'amélioration du réseau routier, par les intendants et la nouvelle administration des Ponts et Chaussées, crée une forte concurrence à la voie d'eau. Le roulage, plus rapide et moins aléatoire que la navigation ligérienne, lui ôte la partie la plus lucrative de son trafic; les voyageurs abandonnent les cabanes, pour des coches plus confortables et à horaires réguliers. De robustes ponts de pierre construits à Nantes, à Tours, à Blois, à Orléans par Perronnet, par Hupeau et par les grands ingénieurs de leur école se substituent aux bacs, aux gués et aux passerelles de bois précaires qui rendaient hasardeux le passage de la ligne d'eau.

La grande sécheresse de 1785 d'abord, puis les guerres de la Révolution et de l'Empire baillent pourtant un répit à la grande marine de Loire: pénurie de chevaux, système routier à l'abandon, littoral bloqué par les croisières anglaises, tout conspire pour un temps en faveur de la batellerie qui connaît un regain d'activité. Les guerres de Vendée et la Chouannerie elles-mêmes en font un boulevard militaire, sillonné par les convois de bateaux armés.

Après 1815, s'esquisse une modernisation du transport par eau dans le bassin ligérien ; on crée des transports accélérés, pour satisfaire - ou tenter de satisfaire - les chargeurs pressés.

La navigation « réglée " prend un certain essor avec le développement de la navigation à vapeur : si le premier vapeur apparaît dès 1820 , les compagnies de bateaux à coques de tôle, à roues et à machine basse pression se multiplient à partir de 1830 et les Concurrents, les Paquebots, les Hirondelles, les Pyroscaphes et autres Inexplosibles se font une concurrence effrénée. Non sans avatars et sans accidents; le Vulcain saute devant Ingrandes en 1837.

Curieusement, le mouvement d'équipement de la Loire s'accélère alors même que les jours de la navigation sont comptés. Et comme il arrive ordinairement en France, où le bon-sens et l'esprit d'à-propos ne sont pas des mieux partagés, c'est lorsque la révolution technique par la machine à vapeur s'affirme, que des crédits considérables sont consentis pour fournir à la grande marine de Loire des moyens nouveaux. Alors qu'Orléans est pourvu d'un capitaine de port (1842), Gien se dote d'un port aux bois et Moulins d'une superbe gare d'eau de 3 hectares de surface utile, qui s'ensablera complètement en quelques années. Selles sur le Cher n'inaugure son beau quai maçonné, pourvu de rampes d'accès et de larges anneaux d'attache, qu'en 1864. D'autres magnifiques quais dallés et de longues cales-abreuvoirs pavées sont alors édifiées dans de médiocres localités riveraines, comme à Bréhémont, en Touraine. En Auvergne, et sur l'Allier, le port de Pont-Du-Château est construit de telle sorte qu'on le nommera par dérision «le Petit Marseille»: mais l'agonie de la grande Marine de Loire, surclassée par le chemin de fer dont le réseau s'étend irrésistiblement, com- 
mence. Et moins de vingt ans plus tard, les Ponts et Chaussées lui portent le coup de grâce en cessant leurs opérations annuelles de balisage et de nettoyage du fleuve.

D'ailleurs, la Loire s'est manifestée de sinistre manière et coup sur coup en 1846, 1856 et 1866 par des inondations désastreuses : d'énormes crues ont ainsi, à dix ans de distance, causé d'énormes dégâts : des dizaines de morts, des scènes d'épouvante, des terroirs fertiles dévastés, de nombreuses maisons englouties, des brèches énormes ouvertes dans les digues par les eaux déchaînées. Bref, un bilan catastrophique qui entraîne une révision déchirante des stratégies adoptées depuis plusieurs siècles pour lutter contre les colères du fleuve. Renonçant à surhausser les digues, et sous l'impulsion de l'ingénieur Comoy, l'administration des ponts et chaussées se borne désormais à entretenir les levées existantes, et établit des déversoirs pour mieux écrêter les crues, tout en organisant une plus stricte surveillance des variations de son débit.

Désormais, significativement, les villes et les campagnes riveraines "tournent le dos " au fleuve ; un fleuve désert, que ne hantent plus guère que les pêcheurs professionnels, de plus en plus rares, ou des amateurs tels que Maurice Genevoix; un fleuve inutile qui paresse entre ses quais déserts ; un fleuve suspect dont la mémoire collective honnit les fureurs, les perfidies, les indolences trompeuses et dans les eaux duquel les baigneurs eux-mêmes ne se risquent qu'avec réticence.

\section{IV 들 DEMAIN LA LOIRE...}

$\mathrm{Y}$ a-t-il un avenir pour ce fleuve au lourd et prestigieux passé ? En cette fin du XX ${ }^{\text {ème }}$ siècle, tout montre que la réponse est oui. Alors que l'eau est promue au rang de bien précieux, qui se valorise d'année en année, les hommes s'intéressent à nouveau au potentiel ligérien. La Loire reprend du service par le truchement du tourisme, de l'économie, de la culture. Convoitée par les uns, fréquentée par d'autres, célébrée et illustrée par beaucoup, elle réoccupe d'ores et déjà une position centrale dans les réalités immédiates, dans les perspectives et les projets à terme court ou long.

L'agence de bassin Loire-Bretagne et l'établissement public pour l'aménagement de la Loire et de ses affluents (EPALA) se penchent sur elle avec sollicitude: la qualité des eaux, la modulation des débits, l'état des rives, la profondeur du chenal, la divagation du lit mineur, les emplois des plans d'eau et les prélèvements de l'agriculture pour irrigation par aspersion, la navigation de plaisance, la baignade, la remontée des poissons migrateurs ou la pêche, la protection des zones inondables bâties ou non bâties... rien ne leur échappe : la Loire est sous tutelle administrative et la vigi- lance est à l'ordre du jour. Vigilance aussi, un peu brouillonne, mais chaleureuse, des ligues écologistes qui veulent - illusion sympathique - conserver « le dernier grand fleuve sauvage d'Europe $»$.

Délaissé, naguère encore, oui. Mais sauvage, que nenni ! Nous espérons l'avoir montré, la Loire, malgré ses foucades, s'est humanisée depuis bien longtemps: loin d'être un obstacle permanent et un milieu répulsif, elle a été un lien entre nos provinces et nos villes; par delà bon nombre de découvertes archéologiques, les vestiges des travaux par lesquels nos ancêtres ont harnaché le fleuve et tous ses affluents sont partout visibles, pour qui sait voir.

- Les centrales nucléaires échelonnées sur les bords de la Loire ;

- Les plans d'eau, aux fins de sports nautiques qui s'y multiplient ;

- Les terrains de camping, faisant fi des crues inopinées ;

- Les prises d'eau au bénéfice d'agriculteurs qui voient dans l'irrigation par aspersion l'ultime chance de leur profession mal aimée ;

- Les bateaux à l'ancienne, toues, chalands ou futureaux qui s'y affrontent en pacifiques compétitions ;

- Les radeaux qui dévalent les gorges écumeuses du haut Allier :

- Les innombrables pêcheurs qui rêvent d'y ferrer le saumon de leur vie, ou sinon quelqu'alose, voire quelque sandre ;

- Les ornithologues qui y observent une pléthore d'oiseaux aquatiques, migrateurs ou sédentaires ;

- Les innovateurs, qui essaient d'y expérimenter des hydroglisseurs commerciaux ;

- Les touristes qui se passionnent pour le riche passé de la vallée, enfin, autant de marques convergeantes et très concrètes de l'intérêt nouveau qui se porte sur cette Loire emblématique, qui fut l'un des liens fédérateurs de la Gaule chevelue et du royaume capétien.

\section{BIBLIOGRAPHIE SUCCINCTE}

[1] VARII AuCtores. - Une histoire de la Loire, Paris Ramsay, 1985.

[2] Dussourd H. - Les hommes de la Loire, Paris, BergerLevrault, 1985.

[3] Mantellier P. - Histoire de la communauté des marchands de Loire et rivières descendant d'icelle, Orléans, 1867.

[4] Poitrineau A. - La Loire, Les Peuples du fleuve, Lyon, Hovath, 1989 . 